\section{MYB bi-allelic targeting abrogates primitive clonogenic progenitors while the emergence of primitive blood cells is not affected}

\author{
Zahir Shah,, ${ }^{1,2,3}$ Elena S. Philonenko, ${ }^{1,2,4}$ Vasily Ramensky, ${ }^{5,6}$ Chenyu Fan,, ${ }^{1,2,3}$ \\ Cuihua Wang,,$^{1,2}$ Hanif Ullah, ${ }^{1,2,3}$ Baoyun Zhang, ${ }^{1,2}$ Pavel Volchkov \\ and Igor M. Samokhvalov ${ }^{1,2}$
}

${ }^{1}$ Guangzhou Institutes of Biomedicine and Health, Chinese Academy of Science, Guangzhou, China; ${ }^{2}$ Key Laboratory of Regenerative Biology, Chinese Academy of Science, Guangdong Provincial Key Laboratory of Stem Cells and Regenerative Medicine, Guangzhou, China; ${ }^{3}$ University of Chinese Academy of Science, Beijing, China; ${ }^{4}$ Vavilov Institute of General Genetics, Russian Academy of Science, Moscow, Russia;

${ }^{5}$ Moscow Institute of Physics and Technology, Dolgoprudny, Moscow, Russia and

${ }^{6}$ National Medical Research Center for Preventive Medicine, the Ministry of Healthcare of the Russian Federation, Moscow, Russia

\section{Ferrata Storti Foundation}

Haematologica 2021

Volume 106(8):2191-2202

\title{
ABSTRACT
}

M $Y B$ is a key regulator of definitive hematopoiesis and it is dispensable for the development of primitive hematopoietic cells in vertebrates. In order to delineate definitive versus primitive hematopoiesis during differentiation of human embryonic stem cells, we have introduced reporters into the MYB locus and inactivated the gene by bi-allelic targeting. In order to recapitulate the early developmental events more adequately, mutant and wild-type human embryonic stem cell lines were differentiated in defined culture conditions without the addition of hematopoietic cytokines. The differentiation of the reporter cell lines demonstrated that MYB is specifically expressed throughout emerging hematopoietic cell populations. Here we show that the disruption of the MYB gene leads to severe defects in the development and proliferation of primitive hematopoietic progenitors while the emergence of primitive blood cells is not affected. We also provide evidence that MYB is essential for neutrophil and T-cell development and the upregulation of innate immunity genes during hematopoietic differentiation. Our results suggest that the endothelial origin of primitive blood cells is direct and does not include the intermediate step of primitive hematopoietic progenitors.

\section{Introduction}

Mammalian hematopoietic development is a multistage process that occurs in two distinct sites of the vertebrate conceptus, the yolk sac and the embryo proper. The primitive hematopoietic program of the yolk sac is transient and restricted to a few blood cell lineages. The earliest clonogenic hematopoietic progenitors arise within the extraembryonic mesoderm of the mid-streak mouse conceptus and precede the appearance of first hemoglobinized erythroblasts by about 1.5 days of gestation. ${ }^{1,2}$ The primitive hematopoietic progenitors are thought to give rise to all primitive erythroblasts and other primitive blood cells. ${ }^{1,3,4}$ The progenitor-derived primitive erythroblasts gradually mature and ultimately enucleate within mouse embryo circulation. ${ }^{5}$

Myb, one of the key hematopoietic transcription factors, is essential for the maintenance of definitive hematopoietic progenitors with high proliferative potential. ${ }^{6}$ In the developmental context, the $M y b$ gene is expressed in the definitive erythroid precursors of mouse fetal liver but is not detected in primitive erythroid cells of the yolk sac. ${ }^{7}$ The homozygous disruption of $M y b$ is embryonic lethal by E15.5 due to progressive anemia caused by defects in the definitive erythropoiesis, whereas primitive hematopoiesis is not affected. ${ }^{8}$ The role of MYB in human hematopoiesis has been studied using the hematopoietic differentiation of human pluripotent stem cells (hPSC) in vitro as a model of human hematopoietic develop-

\section{Correspondence:}

IGOR M. SAMOKHVALOV

igor@gibh.ac.cn

Received: February 4, 2020.

Accepted: July 28, 2020.

Pre-published: July 30, 2020.

https://doi.org/10.3324/haematol.2020.249193

(C)2021 Ferrata Storti Foundation

Material published in Haematologica is covered by copyright. All rights are reserved to the Ferrata Storti Foundation. Use of published material is allowed under the following terms and conditions:

https://creativecommons.org/licenses/by-nc/4.0/legalcode. Copies of published material are allowed for personal or internal use. Sharing published material for non-commercial purposes is subject to the following conditions:

https://creativecommons.org/licenses/by-nc/4.0/legalcode, sect. 3. Reproducing and sharing published material for commercial purposes is not allowed without permission in writing from the publisher. 
ment. These studies have supported the established notion of MYB as the definitive hematopoietic factor that does not contribute to the development of the primitive wave. ${ }^{9,10}$

In an attempt to pinpoint the origin of the definitive hematopoiesis, we generated $M Y B$ reporter and $M Y B$-null human embryonic stem cells (hESC) lines by gene targeting and subjected them to hematopoietic differentiation in defined conditions without exogenous hematopoietic cytokines. Unexpectedly, we have found that MYB is essential for the development and proliferation of primitive clonogenic progenitors. Our results suggest that the early primitive blood cells can develop independently of the primitive clonogenic progenitors that constitute a separate minor cell population of primitive hematopoiesis.

\section{Methods}

The hESC line used in this study was H1 (NIH code WA01). In order to initiate hematopoietic development, briefly formed embryoid bodies (EB) were allowed to attach to surfaces that were coated with extracellular matrix proteins. The cells were differentiated in a Stemline ${ }^{\circledR}$ II SFM (Sigma-Aldrich, St. Louis, MO, USA) based medium supplemented with hVEGF165 (PeproTech, Rocky Hill, NJ). During the first 2 days post-attachment, hBMP4 (PeproTech) was added to initiate mesoderm formation. Additional information on materials and methods is provided in the Online Supplementary Appendix.

\section{Results}

\section{Generation of reporter cell lines and bi-allelic inactivation of MYB}

In order to create $M Y B$ reporter hESC lines, we have introduced alternative fluorescent gene reporters Venus and tdTomato into the second exon of $M Y B$ by TALENmediated homologous recombination (Figure 1A; Online Supplementary Figure $S 1 A$ and $B$ ). The reporter insert containing a strong transcription stop signal has been placed downstream of the transcription elongation attenuation site and the second promoter both located in the first intron. ${ }^{11,12}$ This position of the reporter genes maximized the probability of making reporter expression closely reflect transcription regulation of $M Y B$.

Properly targeted clones were selected by Southern hybridization with two different biotin-labeled probes (Online Supplementary Figure S1C and D). For bi-allelic inactivation, we excised the $P G K-P u r 0^{R}$ cassette by Cre recombinase and subjected resulting Puro ${ }^{S}$ clones to the second round of electroporation with the targeting construct and TALEN (Online Supplementary Figure $1 E$ and F). Real-time reverse transcription polymerase chain reaction (RT-PCR) and western blotting showed that the MYB expression in differentiated bi-allelic knockout cells was effectively switched off (Figure $1 \mathrm{~B}$ and $\mathrm{C}$ ). Analysis of $M Y B$ exonal expression using RNA sequencing of differentiated mutant hESC confirmed that the gene transcription elongation is blocked by the inserted gene cassette (Online Supplementary Figure S2A). The analysis demonstrated that despite the presence of the PGK promoter in the second targeted allele the promoter leakage was negligible. Karyotyping of the targeted cells did not reveal any gross chromosomal aberrations (Online Supplementary Figure S2B).

\section{MYB is specifically expressed in the early human blood cells}

We subjected H1-isogenic hESC reporter lines (single knockout, SKO, cells) and MYB-null lines (double knockout, DKO, cells) to a modified planar hematopoietic differentiation in defined culture conditions. ${ }^{13-15}$ These conditions improve the reproducibility of the data, ${ }^{15}$ which is critical for reliable phenotypic analysis of the mutant hESC lines. Moreover, we did not add hematopoietic cytokines to the differentiation medium for closer recapitulation of the early hematopoietic development. The rationale for adopting such protocol was that high concentrations of hematopoietic cytokines are unlikely to occur in the early conceptus. ${ }^{16}$

The recapitulative quality of such hematopoietic cytokine-free in vitro differentiation was manifested by the spontaneous formation of vascular plexus-like structures and transitory blood island-like VE-Cadherin ${ }^{+}$cell aggregates at which hematopoietic induction occurred in a process similar to the endothelial-to-hematopoietic transition (EHT) (Figure 1D). Single or clumped $\mathrm{CD}^{4} 3^{+}$cells emerged on the fringes of the "blood islands" after apparent downregulation of VE-Cadherin in the peripheral cells, and the loss of VE-Cadherin was followed by dissociation of the nascent blood cells from the aggregates (Figure 1D). The emerging $\mathrm{CD}_{4} 3^{+}$cells induced the expression of key hematopoietic transcription factors, such as GATA1, GATA2, GFI1, GFI1B, KLF1, LMO2, MYB, RUNX1, SPI1, TAL1, which attested the hematopoietic commitment of these cells in contrast to non-hematopoietic CD43-negative cells (Figure 1E). In accordance with the transcriptomics data, MYB-Venus ${ }^{+}$cells emerge within these in vitro blood islands (Figure 1F). Our observations suggest that the segregation of the early blood cells from the hESCderived hemogenic endothelium (HE) is similar to the initiation of hematopoiesis in the yolk sac. ${ }^{2}$

The SKO cells demonstrated vigorous hematopoietic development identical to that of the parent "wild-type" (WT) H1 hESC (Online Supplementary Figure 3A), indicating the absence of non-specific genetic lesions in these cells. Time course quantitation of MYB-Venus fluorescence in SKO cells and MYB mRNA in WT cells showed two peaks of expression, on day 6 and around day 14 (Figure 1G). The close correlation of MYB-Venus flow cytometry of SKO cells and the MYB qRT-PCR data of WT cells indicates that the knockin reporter system faithfully reflects $M Y B$ expression. Undifferentiated SKO hESC were Venus-negative while the MYB-Venus expression was induced upon the upregulation of the earliest hematopoietic markers and concomitant downregulation of pluripotency markers (Figure 2A). Starting on day 6 of hematopoietic differentiation, MYB-Venus was specifically expressed in overlapping blood cell populations (Online Supplementary Figure S3A). Within differentiated cultures on day 6 and day 10, the highest level of MYB-Venus expression was observed in the CD $41 \mathrm{a}^{\text {high }} \mathrm{CD} 235 \mathrm{a}^{+}$erythro-megakaryocyte precursors and the $\mathrm{CD} 34^{\text {low }} \mathrm{CD} 45^{+}$ phenotypic progenitor population, respectively (Online Supplementary Figure S3B and C).

In agreement with the flow cytometry data, RNA sequencing demonstrated around 20 times higher levels of MYB mRNA in day $6 \mathrm{CD} 43^{+}$cells compared to day 6 $\mathrm{CD}^{-} 3^{-}$non-blood cells (Figure $2 \mathrm{~B}$ ). The $\mathrm{MYB}^{+} / \mathrm{CD}^{4} 3^{+}$cells also selectively expressed high levels of GYPA (CD235a) and ITGA2B (CD41a) mRNA. These markers were shown 
A

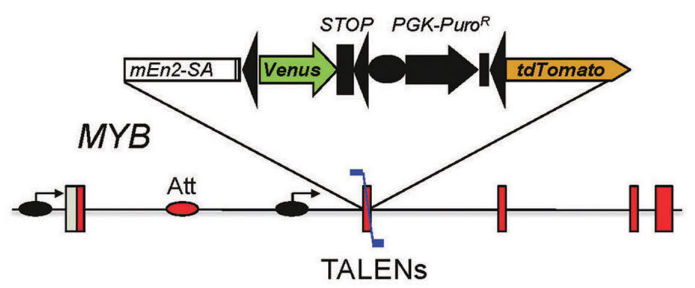

D

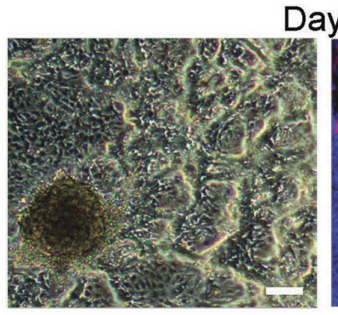

Day 5

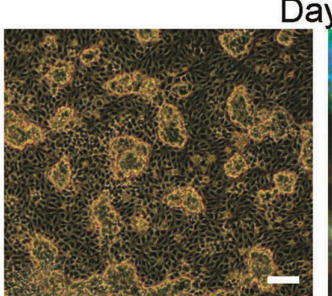

Day 6

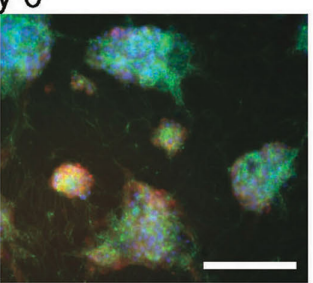

Day 8
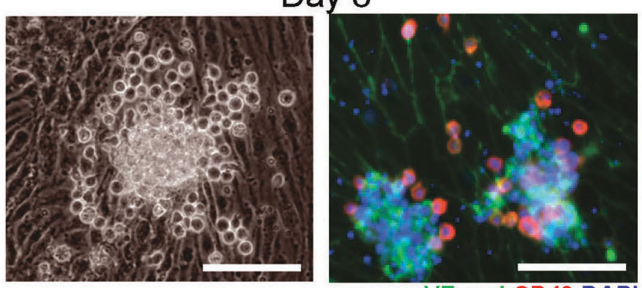

VE-cad CD43 DAPI

F

Day 7

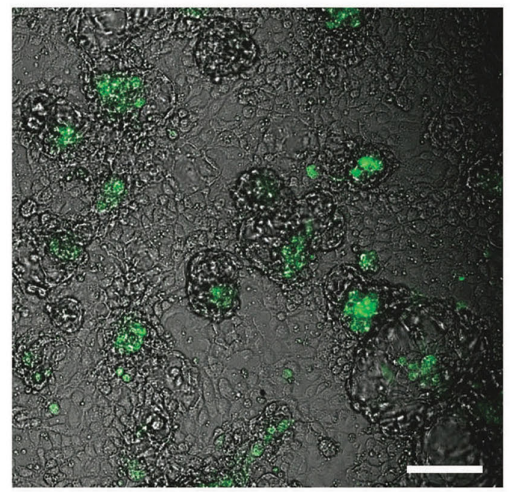

MYB-Venus

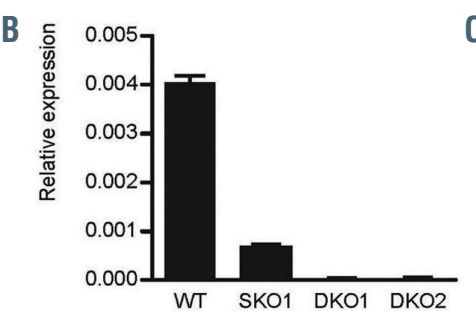

C

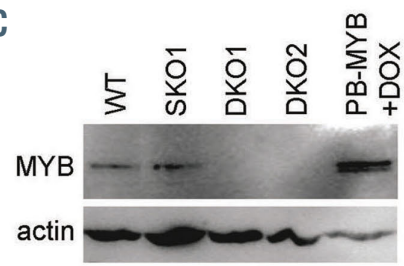

E

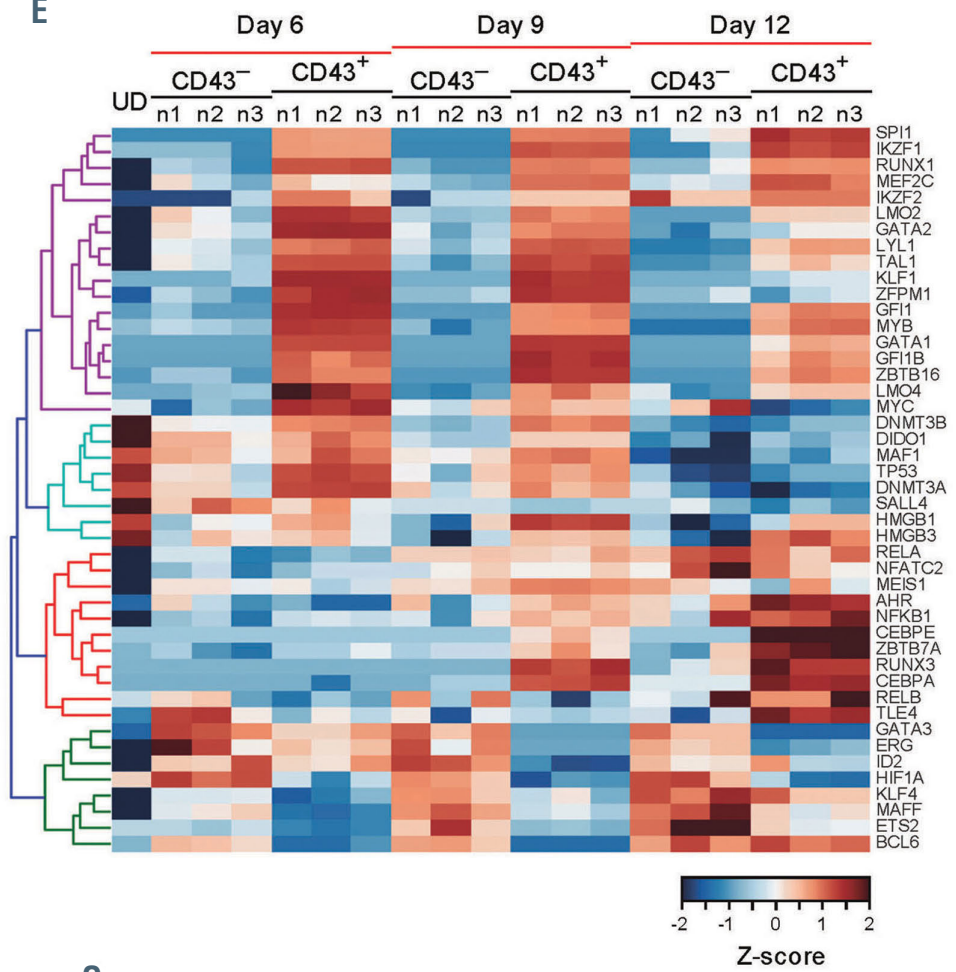

G
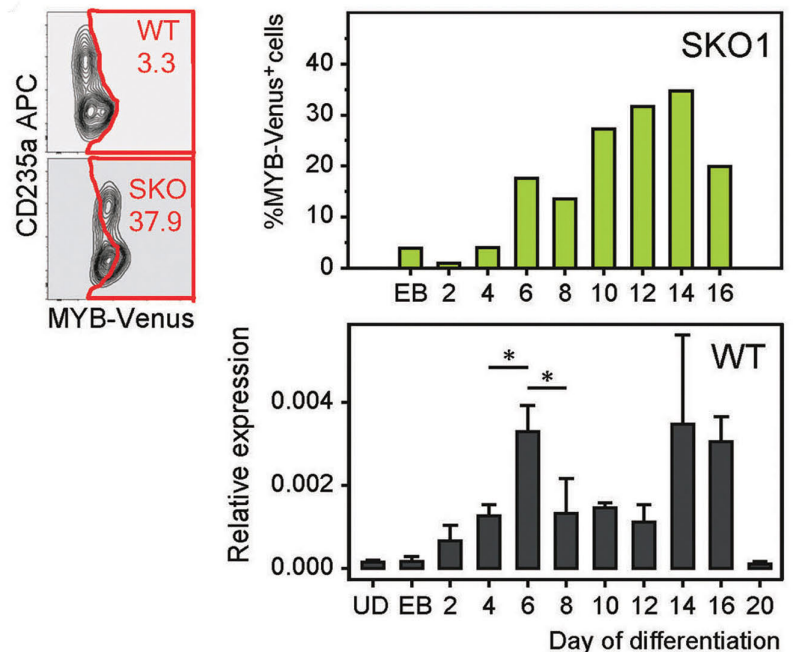

Figure 1. The outline of the experimental system for studying the role of MYB in early human hematopoietic development. (A) Scheme of the MYB gene targeting. Designations are as in Online Supplementary Figure S1B. (B) Quantitative real-time polymersase chain reaction (qRT-PCR) analysis of the MYB expression in day 6 differentiated wild-type (WT), SKO, and DKO cells. Here and elsewhere: SKO1, DKO1, and DKO2, independent single and double knockout H1-isogenic cell lines. (C) Western blot analysis of day 12 differentiated MYB knockout cells. PB-MYB+DOX, MYB-null cells transfected with a PiggyBack-TRE-CMV-MYB cDNA vector and activated by doxycycline (DOX). (D) In vitro blood islands in the cytokine-free hematopoietic differentiation of human embryonic stem cells (hESC). Upper panels show representative phase-contrast images of the early stages of $\mathrm{H} 1 \mathrm{hESC}$ differentiation, the lower panels - immunocytofluorescent staining of the cultures at the corresponding stages of differentiation. Scale bar - $100 \mu \mathrm{m}$. (E) Expression of selected transcription factors in $\mathrm{CD}_{43^{+}}$versus $\mathrm{CD}^{-} 3^{-}$cell population on day 6,9 , and 12 of hematopoietic differentiation of $\mathrm{H} 1 \mathrm{hESC}$. Gene expression levels in the heatmap are normalized by Z-score transformation across the RNA-seq experiments, with three independent biological repeats for each cell population. $(F)$ The emergence of MYB-Venus ${ }^{+}$DKO cells (DKO2) from the in vitro blood island structures on day 7 of differentiation. Scale bar - $100 \mu \mathrm{m}$. (G) Venus reporter expression faithfully reflects the dynamics of MYB mRNA levels during the time course of differentiation. The upper bar plot is the quantitation, exemplified by contour plots on the left, of the MYB-Venus expression dynamics in SKO cells (Online Supplementary Figure S3A). The lower bar plot demonstrates the quantitation of MYB mRNA by qRT-PCR in undifferentiated (UD) and differentiated WT H1 hESC on the EB stage (day 0) through day 20 . The data are mean \pm standard deviation, ${ }^{*} P<0.05$, two-tailed Student's $t$-test. 
to be specific for the hESC-derived primitive human hematopoiesis. ${ }^{17,18}$ Correspondingly, cell populations expressing CD43, CD235a, and CD41a were strongly suppressed by SB-431542, the inhibitor of Activin/Nodal signaling and primitive human hematopoiesis ${ }^{17}$ (Figure 2C). Principal component analysis (PCA) revealed a very close similarity of gene expression profiles in day $6 \mathrm{DKO} / \mathrm{SKO}$ MYB-Venus ${ }^{+}$cells and WT CD $43^{+}$primitive blood cells whereas gene expression of day $6 \mathrm{DKO} / \mathrm{SKO}$ MYB-Venus cells closely correlated with the profile of WT CD43- cells (Figure 2D). Taken together, these observations indicate that in contrast to mouse data ${ }^{1,7}$ MYB is specifically expressed in the primitive human blood cells.

\section{Defective development of myeloid and T cells derived from MYB-null human embryonic stem cells}

The MYB-Venus fluorescence in differentiated DKO hESC was much stronger compared to SKO cells, while the hematopoietic specificity of the reporter expression was preserved (Figure 2E). The upregulation of MYB-Venus expression in the MYB-null cells was significantly higher than the expected two-fold rise in cells with the bi-allelic knockin of a reporter gene (Figure 2F) indicating negative transcriptional autoregulation of MYB transcription during the early human hematopoietic development.

Despite the specific expression of MYB in early human blood cells, its bi-allelic inactivation did not perturb the emergence of blood cells at the early stages of differentiation (Figures 2E and 3A). The vast majority of these MYB-independent early blood cell populations were CD235a $\mathrm{a}^{+} / \mathrm{CD} 41 \mathrm{a}^{+}$cells of the primitive erythromegakaryocytic lineage (Figure 3A). Minor qualitative alterations in the expression of hematopoietic markers were noticeable in the $\mathrm{CD} 43^{+} \mathrm{CD} 45^{+} \mathrm{DKO}$ cell population starting from day 10 of differentiation. (Figure $3 \mathrm{~B}$ ). The phenotypical differences become increasingly prominent by day 12 (Online Supplementary Figure 4A). By day 20, DKO cells failed to develop into $\mathrm{CD} 14^{\text {low }} \mathrm{CD} 66 \mathrm{~b}^{+} \mathrm{CD} 86^{-}$ neutrophil granulocytes, and the development of $\mathrm{CD} 11 \mathrm{~b}^{+}$ immature myeloid cells was also negatively affected (Figure 3C). SKO cells demonstrated an intermediate myeloid phenotype, indicating a dose-dependent manner of MYB contribution to early human myeloid development and further validating the observed phenotype of MYB-null cells. The emergence of $\mathrm{CD} 14^{+} \mathrm{CD} 86^{+}$monocytes and macrophages was apparently independent of MYB. The defect in the myeloid development of DKO cells was detected already on day 16 (Online Supplementary Figure S4B). Thus, MYB does not participate, or its deficiency is compensated, in the initial hematopoietic commitment, but the gene is critically important for granulocyte development and maturation.

The transcriptomes of MYB-Venus ${ }^{+} / \mathrm{CD} 43^{+}$early blood cell populations sustained significant changes between day 6 and day 12. In contrast, the gene expression profiles of non-blood MYB-Venus/CD43- remained almost the same (Figure 2D), except day 12 SKO MYB-Venus populations containing blood cells that by day 12 further decreased the low MYB-Venus fluorescence of SKO cells and were sorted into the MYB-negative population. The day 6 - day 12 shift in gene expression of MYBVenus ${ }^{+} / \mathrm{CD} 3^{+}$cells reflects a vigorous hematopoietic development in which MYB had limited influence because day $12 \mathrm{DKO} / \mathrm{SKO}$ MYB-Venus ${ }^{+}$cells continued to cluster with day $12 \mathrm{WT} \mathrm{CD43}{ }^{+}$cells. The data support our phenotypic observations that the functional influence of MYB on the emergence of early blood cells is limited.

Nevertheless, the transcriptomes of DKO/SKO MYB-Venus ${ }^{+}$and WT CD43 ${ }^{+}$cell populations were more divergent by day 12 compared to the close clustering of day 6 populations (Figure 2D). This divergence was sufficient for a reliable differential gene expression (DEG) analysis, and the GO term enrichment revealed a clear pattern of expression changes that occurred in MYB-null blood cells. Inactivation of MYB resulted in the downregulation of genes responsible for the innate immune and inflammatory response (Figure 4A; Online Supplementary Figure S5A). In DKO cells, mainly non-hematopoietic genes were upregulated, which suggests that MYB disruption switches hESC differentiation from hematopoietic to non-hematopoietic development. We also found that in addition to known MYB target genes such as GFI1, and $\mathrm{BCL} 2$, the MYB deficiency led to the suppression of two key regulators of myeloid differentiation, CEBPA and SPI1 (PU.1) (Figure 4B). Furthermore, the expression of CDK6, a key regulator of HSC activation, ${ }^{19}$ was inhibited in DKO cells whereas several CDK inhibitors were upregulated including CDKN1A (CIP1/WAF1), a major mediator of p53-dependent tumor suppression. ${ }^{20}$ The expression of myeloid cytokine receptor genes, CSF1R and CSF3R, as well as a number of genes encoding receptors for pro- and anti-inflammatory cytokines were negatively affected by MYB inactivation (Online Supplementary Figure 5B). The expression of CSF3R was most severely affected, which can explain the observed defects in the granulocyte development of MYB-null cells. In sum, the transcriptome profiling confirmed the involvement of MYB in the development of innate immune myeloid cells and indicated possible mechanisms of MYB regulation of the early human hematopoiesis.

We also confirmed the crucial role of MYB in the development of definitive hematopoiesis. Analysis of the T-cell potential is a standard procedure to monitor definitive hematopoietic development in differentiating cultures of hPSC. ${ }^{17}$ We found that MYB inactivation led to complete failure of lymphoid cell development in the co-culture with OP9-DL4 stroma while WT cells efficiently produced $\mathrm{CD}^{+} \mathrm{CD}^{+}$and $\mathrm{CD}^{+} \mathrm{CD}^{+} \mathrm{T}$ cells (Online Supplementary Figure S6).

\section{MYB inactivation results in the deficiency of primitive clonogenic progenitors}

Next, we investigated whether MYB is required for the development of clonogenic hematopoietic progenitors. In our differentiation system, the earliest clonogenic progenitors arise on day 6 , and their emergence coincides with the spontaneous formation of the in vitro blood islands (Figure 1D). All erythroid/erythro-myeloid progenitors corresponded to the primitive hematopoietic lineage producing primitive erythroblasts of characteristic morphology and primitive megakaryocytes distinguished by their relatively smaller size and less lobular nuclei (Figure 5A). ${ }^{21}$ Moreover, gene expression profiling of pooled methylcellulose colonies showed the overwhelming predominance of embryonic hemoglobin expression (Figure $5 \mathrm{~B})$. We designated the hESC-derived primitive erythroid progenitors with higher proliferative potential as BFU-E ${ }^{P}$ (Burst Forming Unit - Erythroid, Primitive), and those with low proliferative potential as CFU-E ${ }^{\mathrm{P}}$ (Colony Forming Unit - 
A
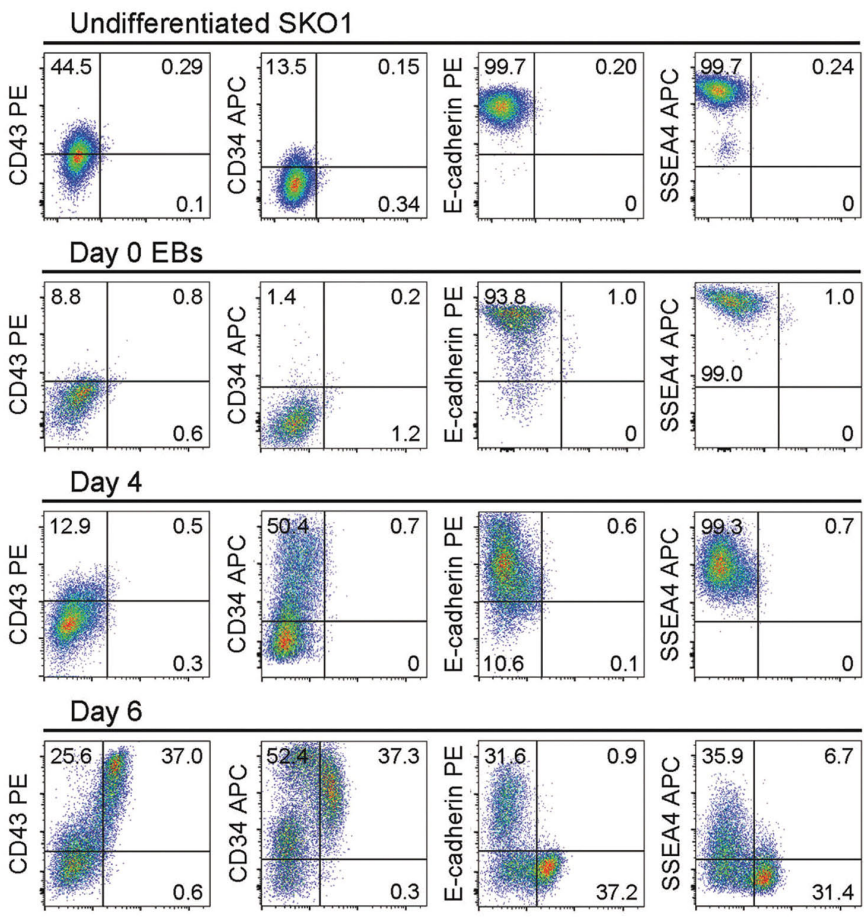

MYB-Venu

0.3
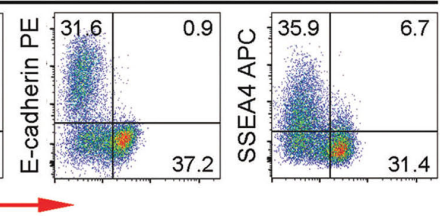

D

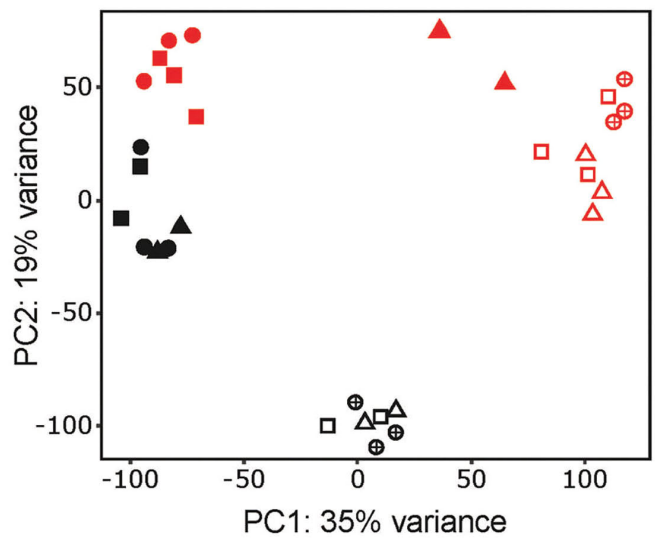

Day 6

口 DKOVenus ${ }^{+}$

$\Delta$ SKOVenus $^{+}$

$\oplus$ WT CD43 ${ }^{+}$

- DKOVenus

A SKOVenus-

- WT CD43-

Day 12

口 DKOVenus ${ }^{+}$

$\triangle$ SKOVenus $^{+}$

$\oplus{\mathrm{WT} \mathrm{CD} 43^{+}}^{+}$

- DKOVenus -

$\triangle$ SKOVenus $^{-}$

- WT CD43-

PC1: $35 \%$ variance

E

Day 10 non-adherent cells
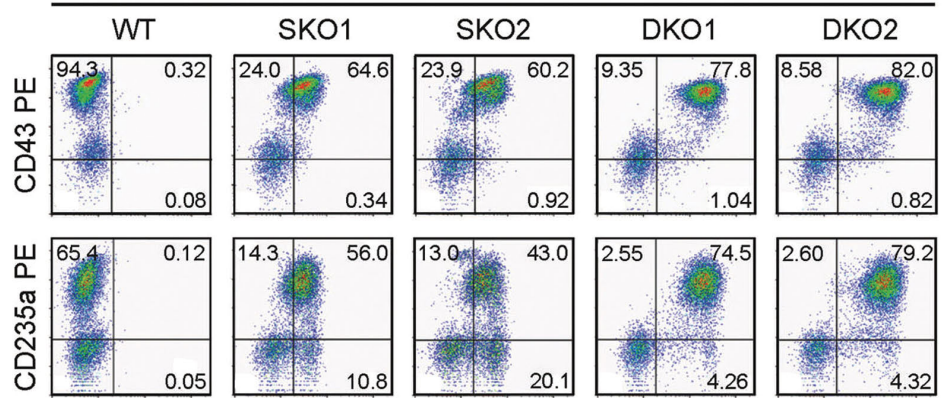

B

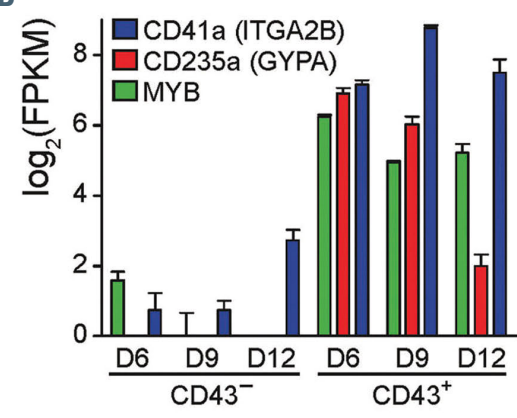

C

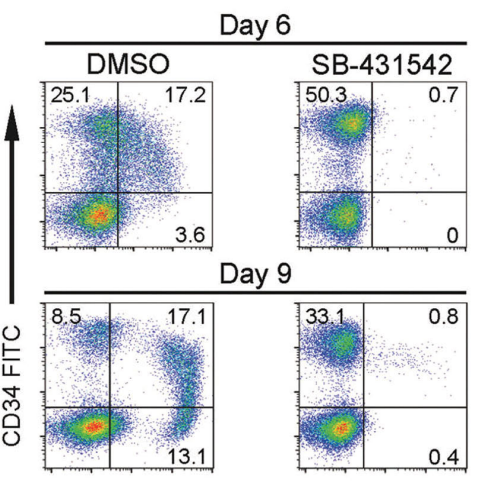

CD43 APC

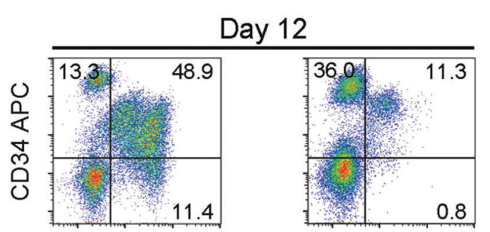

CD43 FITC

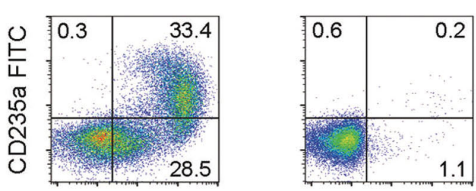

CD41a APC

F

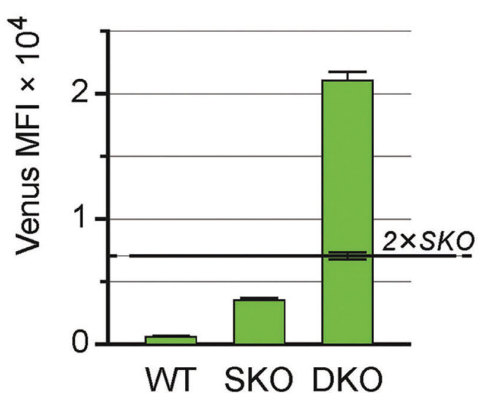

Figure 2. MYB is specifically expressed in the emerging hematopoietic cells. (A) Specific expression of MYB-Venus in the early blood cells during the initial phase of hematopoietic differentiation of SKO1 cells. Here and elsewhere, numbers in flow cytometry plots represent the percentages of cells within the respective quadrants. Representative flow cytometry data of four experiments are shown. (B) Human embryonic stem cells (hESC)-derived CD43 ${ }^{+}$cells selectively co-express mRNA of MYB and the markers of primitive human blood cells, ITGA2B and GYPA. Differentiated wild-type (WT) H1 hESC were used for transcriptome analysis. (C) Inhibition of Activin/Nodal signaling by addition of $6 \mu \mathrm{M}$ SB-431542 between day 2 and day 4 of differentiation strongly suppressed the development of CD43+ and

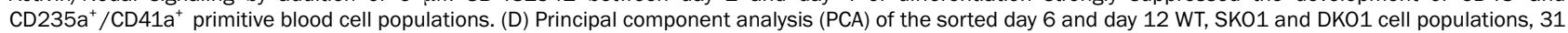
samples, and entire gene set (19,796 genes). (E) Representative flow cytometry of day 10 live non-adherent blood cells generated by $\mathrm{H} 1$ and the isogenic mutant cell lines. (F) Quantitation of data represented in (E). MYB-Venus fluorescence (expressed as the mean fluorescence intensity [MFI]) in DKO cells is disproportionally stronger than the fluorescence in SKO cells. Data are mean \pm standard deviation, $n=4$. 
A

Day 6

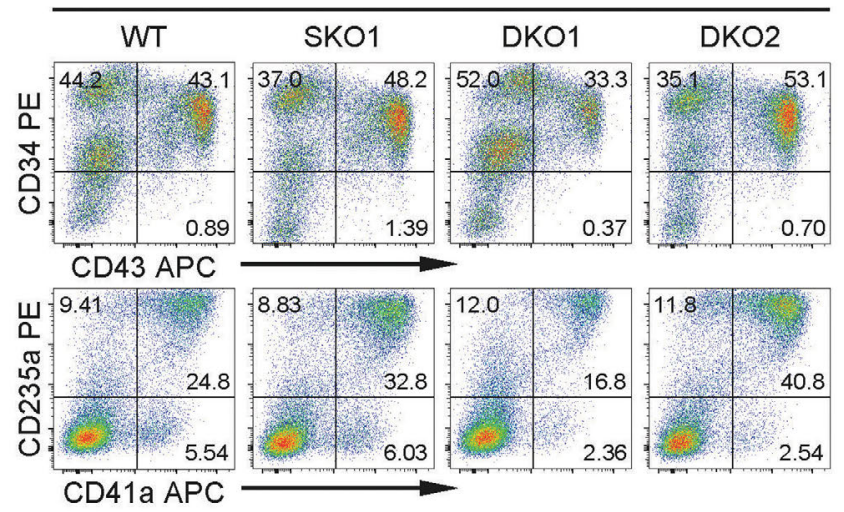

B

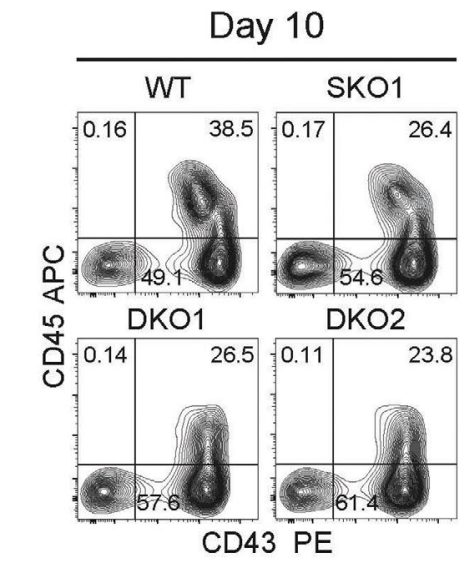

C

Day 20

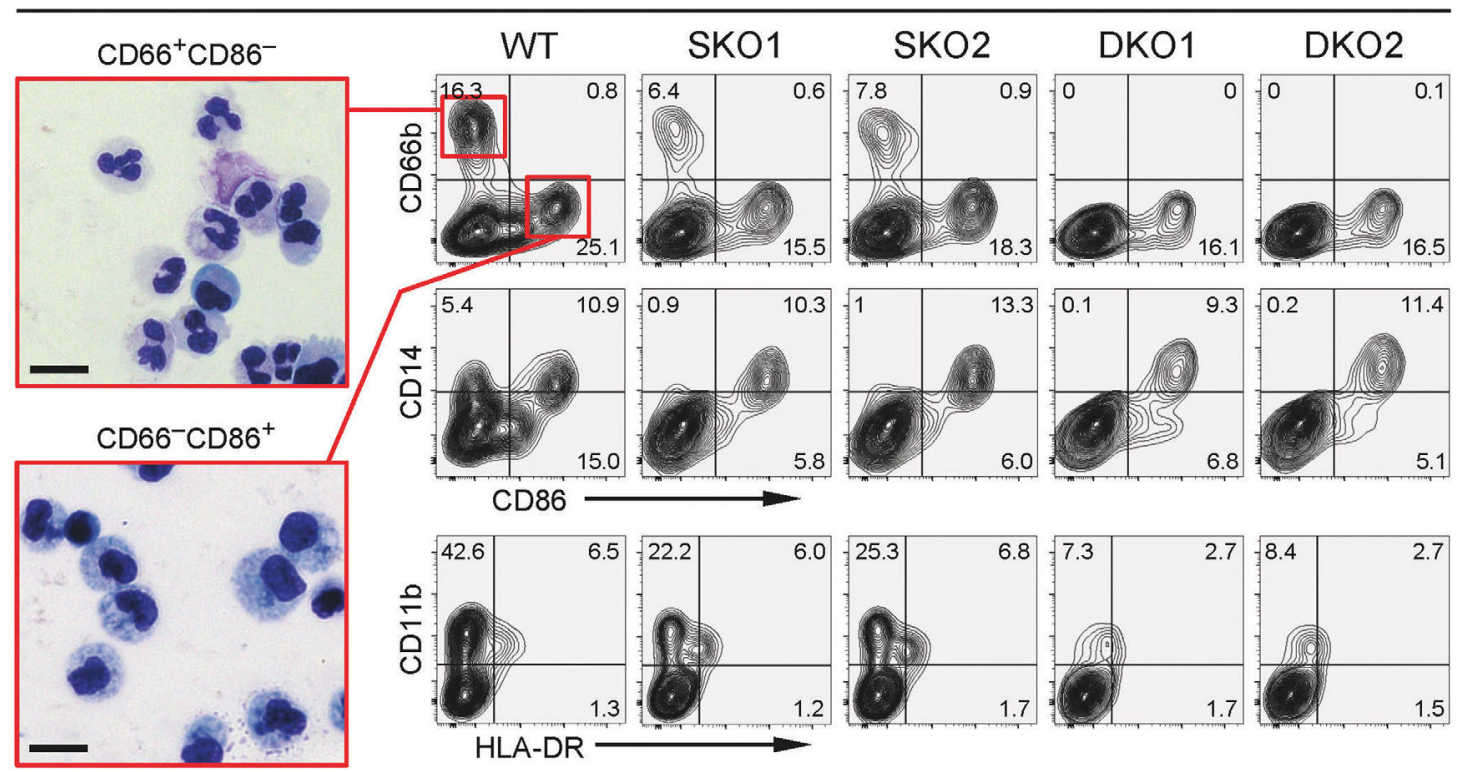

Figure 3. Blood cell populations are differently affected by the MYB deficiency depending on the stage of differentiation. (A) H1-isogenic MYB knockout cell lines develop normally on day 6 of differentiation. Total live differentiated cells were taken for the analysis, as in (B) and (C). Representative data of more than ten experiments are shown. (B) On day 10 the DKO cell lines demonstrate subtle differences in the CD43/CD45 cell staining. Representative data of three experiments are shown. (C) Day 20 phenotype of the MYB knockout cell lines. Representative data of six experiments are shown. Two cytospin panels on the left represent MayGrünwald staining of sorted $\mathrm{CD} 66 \mathrm{~b}^{+} \mathrm{CD} 86^{-}$neutrophils and $\mathrm{CD} 66 \mathrm{~b}^{-} \mathrm{CD} 86^{+}$cells of the monocyte-macrophage lineage. Scale bar, $10 \mu \mathrm{m}$.

Erythroid, Primitive). The ability of the primitive progenitors to form large colonies of erythroid cells suggests that the assay medium is optimal for analyzing primitive progenitors generated in the cytokine-free differentiation.

First, we determined that the vast majority of hematopoietic progenitors were MYB-Venus ${ }^{+}$(Figure 5C). Of note, the analysis of the sorted cell populations demonstrated extremely high efficiency of hematopoietic progenitor generation in our differentiation system: up to $17 \%$ of all day $6 \mathrm{CD} 4^{+} \mathrm{MYB}-\mathrm{Venus}^{+}$blood cells generated CFU-C colonies in the assay medium. Then, we compared the generation of the progenitors by WT and mutant hESC lines on day 6 and day 10 of differentiation. Progenitors of higher proliferative potential, BFU- $E^{\mathrm{P}}$ and CFU-Mix ${ }^{\mathrm{P}}$, were practically absent in differentiated cultures of MYB-null hESC, and the myeloid progenitors were also affected (Figure 5D). Furthermore, we observed the effects of $M Y B$ haploinsufficiency on the generation of these progenitors from SKO cells. Only day 6 erythroid progenitors of low proliferation potential, CFU- $E^{\mathrm{P}}$, could be detected at a comparable density in the MYB-null cultures. However, CFU-E $\mathrm{E}^{\mathrm{P}}$ in day 6 non-adherent $\mathrm{DKO}$ cell fractions were completely lost (Figure 5D). Moreover, instead of forming typical compact cell colonies, nearly all of day 6 MYB-null CFU-E $\mathrm{E}^{\mathrm{P}}$ generated aberrant loose colonies, and the morphology of cells from those colonies was abnormal (Figure 5E). Almost all CFU-myeloid colonies generated by the DKO cells were composed exclusively of macrophages and were notably smaller compared to the WT and SKO counterparts (Figure 5F). The survival of the CFU-M subset of CFU-myeloid progenitors confirms the previously published data on MYBindependence of hPSC-derived macrophages and macrophage progenitors. ${ }^{10}$ Taken together, these observations demonstrate that primitive erythroid and primitive mixed progenitors are non-functional in differentiated cul- 
A

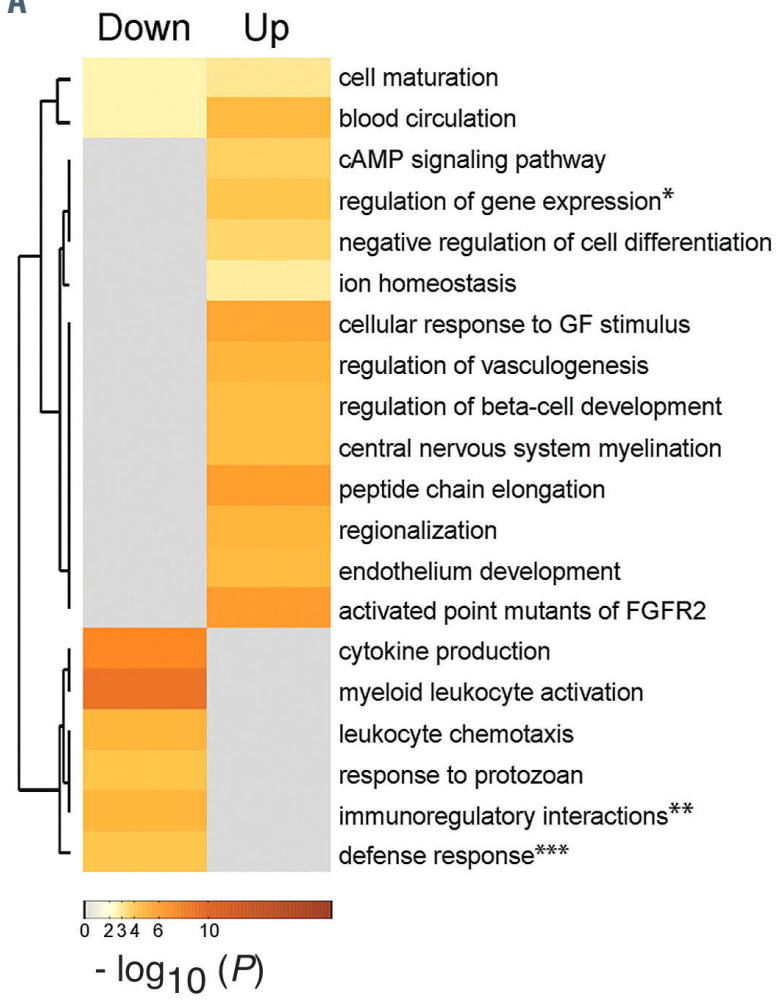

B

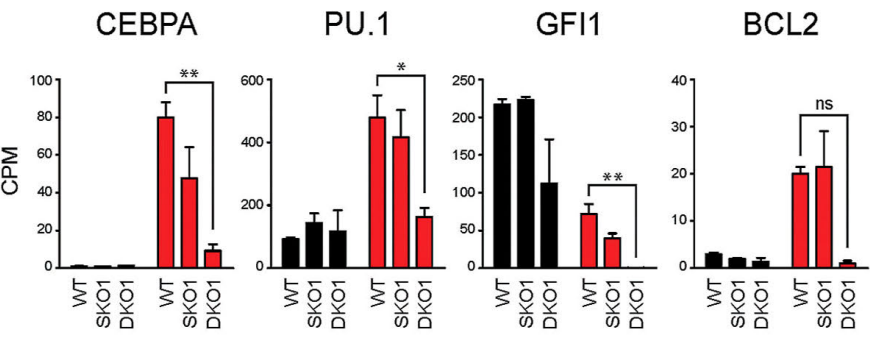

CDK6 CDKN1A CDKN1B CDKN2D

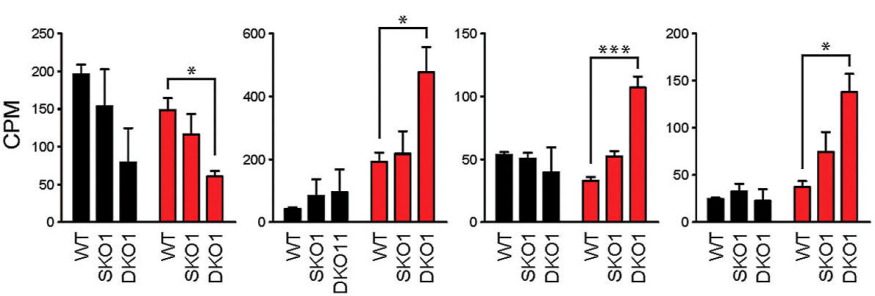

Figure 4. Innate immunity genes, hematopoietic transcription factors, and cell cycle regulatory genes negatively regulated in MYB-null cells. (A) Heat map of top 20 enriched terms across the two differential gene expression (DEG) lists (upregulated and downregulated, log ${ }_{2} \mathrm{FC}>2,<-2$ ) in MYB-null cells, colored by $P$-values. *

- R-HSA-210747: "regulation of gene expression in early pancreatic precursor cells"; ** - R-HSA-198933: "immunoregulatory interactions between a lymphoid and a non-lymphoid cell”; $* * *$ - GO:0098542: "defense response to other organism". (B) Bar plots of mRNA expression of cell cycle regulatory genes and hematopoietic transcription factors in wild-type (WT) CD43 ${ }^{+}$, and MYB-Venus ${ }^{+}$SKO and DKO cells. Data are mean \pm standard error of the mean (SEM), $n=3$ (biological repeats). $P$ values were calculated using one-way ANOVA with Tukey's test for multiple comparisons, where $* * * P<0.001 ; * * P<0.01 ; * P<0.05$.

tures of MYB-null hESC whereas non-macrophage myeloid progenitors are noticeably affected by the MYB inactivation. Such results are unexpected since previous studies denied any role of Myb/myb/MYB in primitive hematopoiesis. ${ }^{7-9,22}$ It was reported earlier that BFU-E and CFU-Mix progenitors failed to develop from Myb-null mESC, although whether such progenitors belonged to primitive or definitive hematopoiesis was unclear. ${ }^{23}$

In order to validate our discovery that MYB was required for the development of primitive human hematopoietic progenitors, we stimulated hESC-derived primitive hematopoiesis Activin A and studied the effects of such treatment on the hematopoietic differentiation of WT, DKO, and SKO cells. Primitive, but not definitive, human hematopoiesis is stimulated by Activin/Nodal signaling. ${ }^{17,24}$ In our experiments, the Activin A treatment led to a reliable increase of MYB-Venus expression in both SKO and DKO blood cells (Figure 5G) and a significant expansion of all types of day 6 clonogenic progenitors derived from the WT hESC (Figure 5H). The latter observation further indicated that all these progenitors were primitive. As expected, MYB-null cells failed to boost the generation of BFU- $\mathrm{E}^{\mathrm{P}}$ and $\mathrm{CFU}-\mathrm{Mix}{ }^{\mathrm{P}}$ progenitors upon the Activin stimulation. The stimulation of $\mathrm{CFU}-\mathrm{E}^{\mathrm{P}}$ was not, however, precluded by the bi-allelic inactivation of MYB, although these MYB-null progenitors formed substandard colonies as described above. Activin-stimulated DKO myeloid progenitors formed only macrophage colonies confirming the resistance of the macrophage lineage to
MYB inactivation. ${ }^{10}$ These results confirm that MYB is required for the development and/or proliferation of primitive human hematopoietic progenitors.

\section{VEGF is a major mitogen of early human hematopoiesis}

Our results demonstrated that primitive blood cells emerged normally in the circumstances, at which primitive hematopoietic progenitors were severely compromised. Apparent progenitor-independence of the early blood cells poses a question of what factors drive the expansion of the early hematopoiesis.

VEGF is the only growth factor that was added to the culture medium after mesoderm induction and, therefore, it is an obvious candidate for a stimulatory molecule promoting the emergence and expansion of early blood cells. Indeed, the removal of VEGF from culture medium starting from day 3 of the differentiation timecourse led to a strong inhibition, but not a complete suppression, of the early hematopoiesis (Figure 6A), which indicates that this growth factor is responsible for mitogenic support of early human blood cells. In conjunction with the resistance of the early blood cells to MYB inactivation, these observations strongly suggest that MYB and VEGF regulate early human hematopoiesis independently. The VEGF removal also abolished the generation of clonogenic progenitors but did not prevent the robust generation of myeloid cells at the later stages (Figure $6 \mathrm{~B}$ to D). The progenitor-independent myeloid cells do not originate from the MYB- 
A

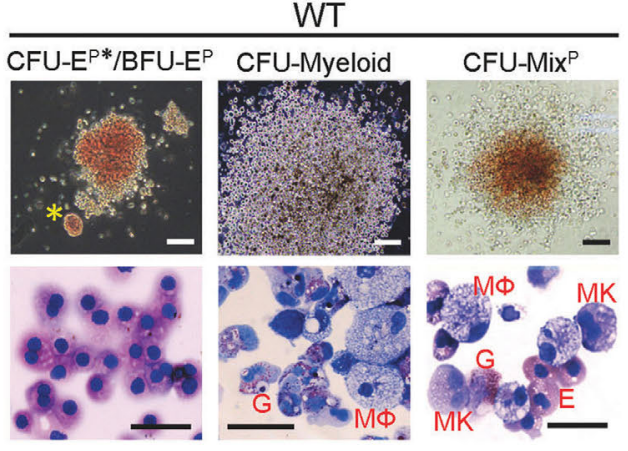

B

Day 6

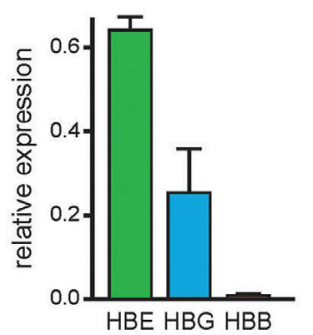

Day 12

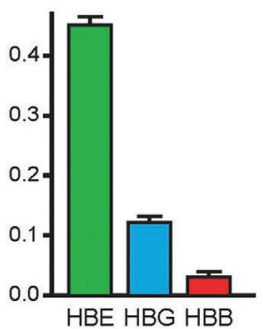

C

SKO1 CD34+
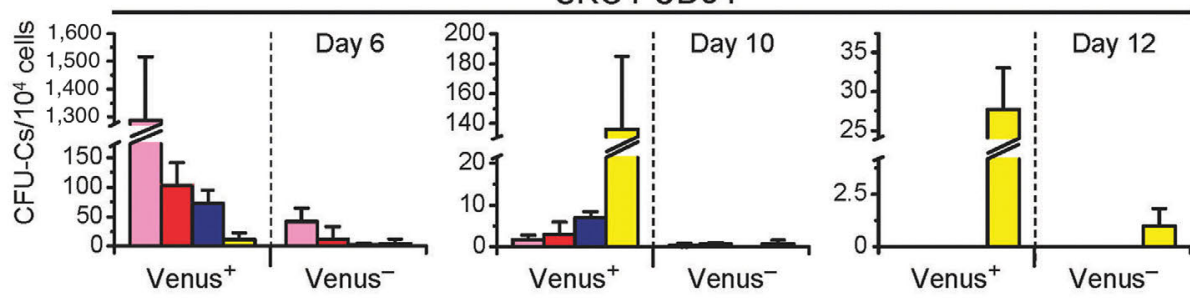

$\square$ CFU-E ${ }^{P}$

BFU-E

$\square$ CFU-Myeloid

$\square$ CFU-Mix ${ }^{P}$

D

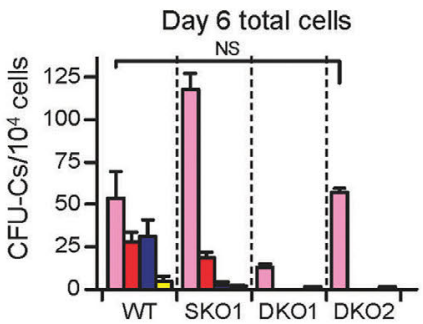

Day 6 non-adherent cells

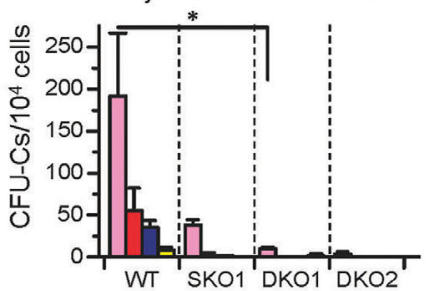

Day 10 total cells

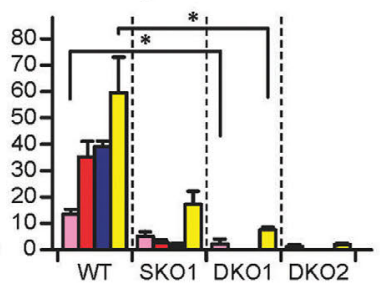

Day 10 non-adherent cells

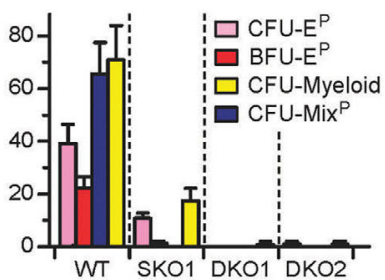

E

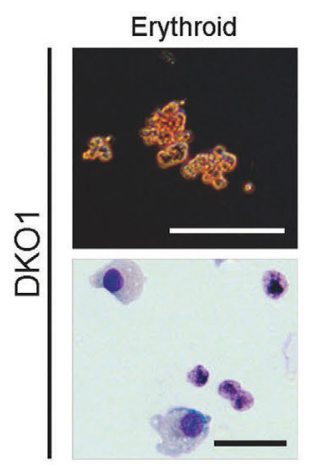

F

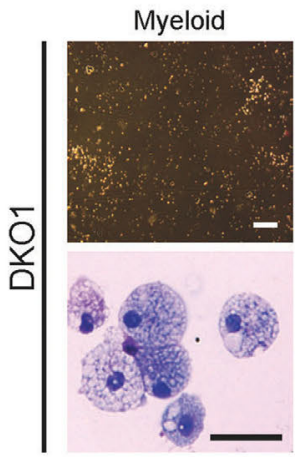

G

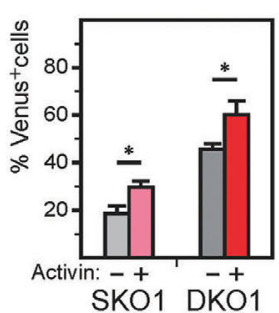

H

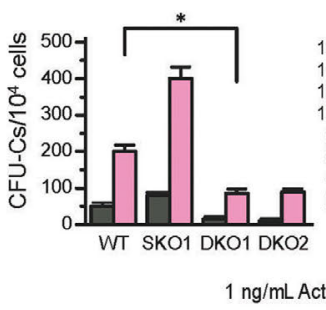

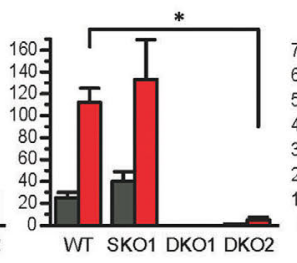

BFU-E $E^{p}$

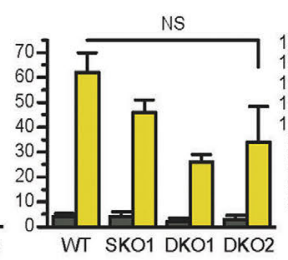

180
160
140
120
100
80
60
40
20
02

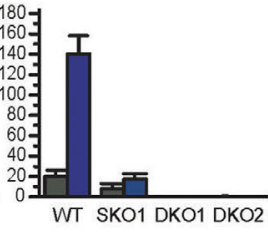

Figure 5. MYB inactivation leads to a severe deficiency of primitive clonogenic progenitors. (A) Morphology of individual CFU-C colonies derived from day 6 and day 10 hematopoietic progenitors (upper panels; scale bar, $100 \mu \mathrm{m}$ ), and May-Grünwald staining of cells from corresponding colonies (lower panels; scale bar, 20 um). MФ: macrophages; MK: megakaryocytes; E: erythroblasts; Gr: granulocytes. (B) Real-time polymerase chain reaction (RT-PCR) analysis of hemoglobin gene expression. Differentiated $\mathrm{H} 1$ embryonic stem cells (ESC) $\left(1 \times 10^{4}\right.$ cells) were grown in methylcellulose assay medium for 18 days, and all resulting CFU-C colonies ( 100150) were pooled for mRNA isolation. The data are mean \pm standard deviation (SD). (C) Primitive human clonogenic progenitors express MYB. The frequency of the hematopoietic progenitors was measured in SK01 CD34 ${ }^{+}$Venus ${ }^{+}$versus CD34 ${ }^{+}$Venus ${ }^{-}$cells. Data are mean \pm SD, $n=3$. (D) The frequency of clonogenic hematopoietic progenitors in total or non-adherent cells of the three H1-isogenic MYB genotypes. Mean values \pm SD are shown, $n=3 . * P<0.05$; NS: non-significant; two-tailed Student's $t$-test. (E) Morphology of typical DKO erythroid colonies and cells. Upper panels, scale bar - $100 \mu \mathrm{m}$; lower panels, scale bar - $20 \mu \mathrm{m}$. (F) Morphology of typical DKO myeloid colonies and cells. Scale bar $-20 \mu \mathrm{m}$. (G) Activin stimulates MYB-Venus expression in the MYB knockout cell lines on day 6 of differentiation. Data are mean $\pm \mathrm{SD}, \mathrm{n}=4$. ${ }^{*} P<0.05$; two-tailed Student's $t$-test. $(\mathrm{H})$ Activin stimulation of day 6 primitive clonogenic progenitors of the three $\mathrm{H} 1$-isogenic $M Y B$ genotypes. Data are mean $\pm \mathrm{SD}, \mathrm{n}=3$. ${ }^{*} P<0.05$; NS: non-significant; two-tailed Student's $t$-test. 
A

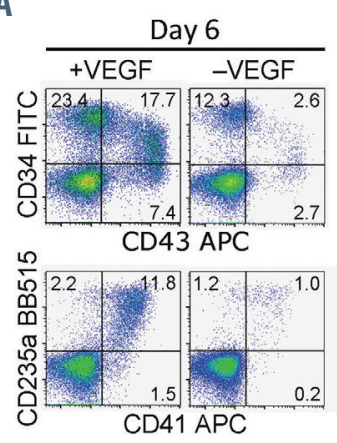

B

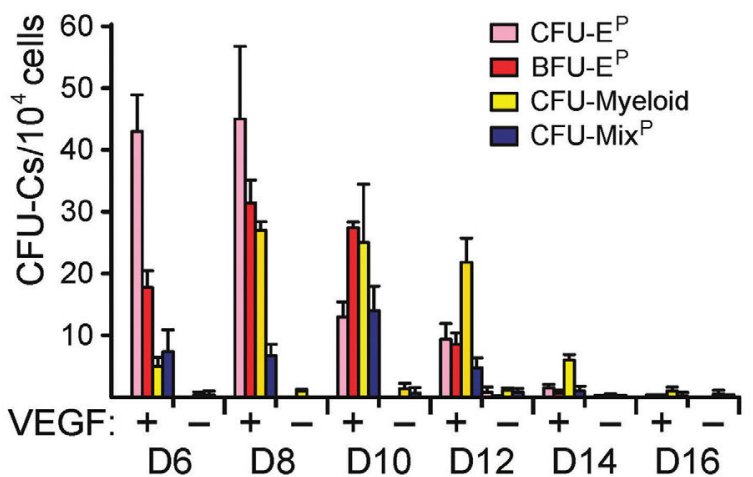

C
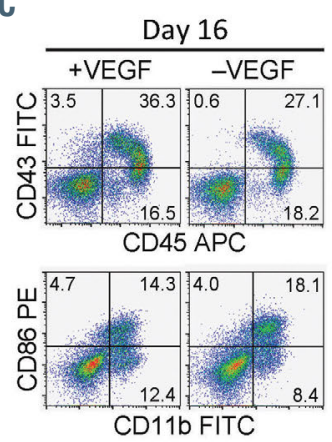

D

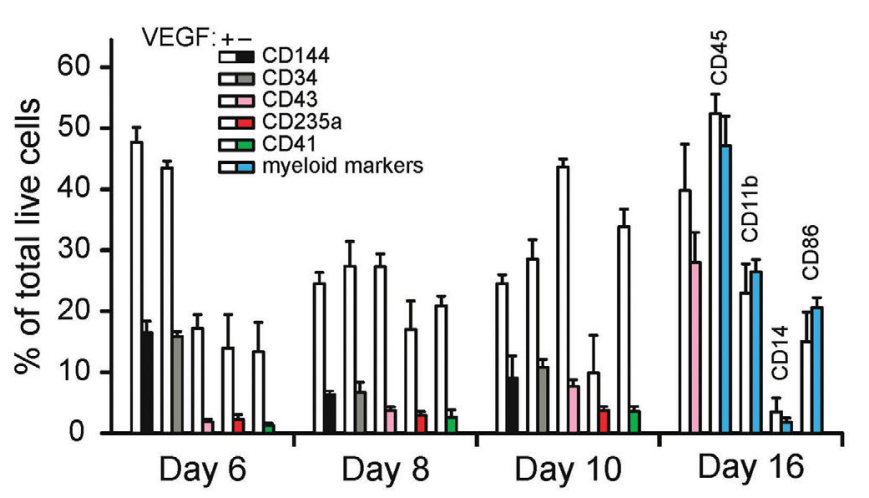

E no VEGF Day 20

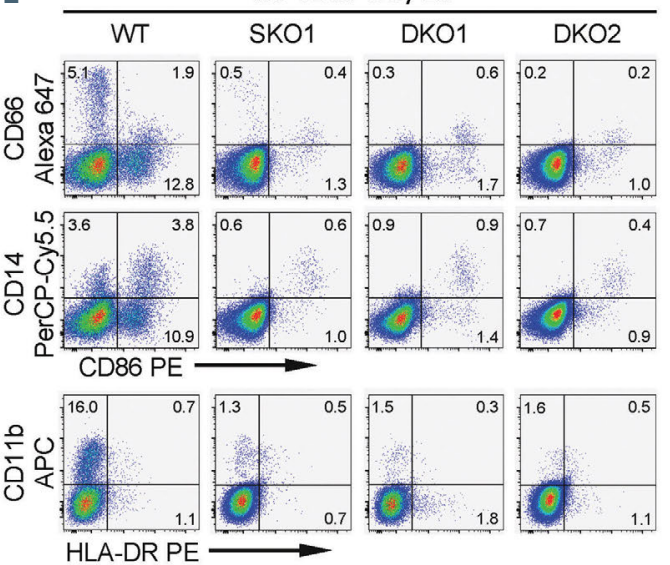

Figure 6. Vascular endothelial growth factor is a key cytokine for the early human hematopoiesis. (A) Human embryonic stem cells (hESC)-derived hematopoiesis on day 6 of differentiation is severely inhibited upon removal of vascular endothelial growth factor (VEGF) starting from day 3 . Representative data of three experiments are shown. (B) Effect of the VEGF removal on the emergence of hematopoietic clonogenic progenitors during differentiation of H1 hESC (day 6 - day 16). Data are mean \pm standard deviation (SD), $n=4$. (C) hESC-derived myeloid cells emerge normally on day 16 of differentiation in the absence of exogenous VEGF. Representative data of four experiments are shown. (D) Dynamics of hematopoietic marker expression during $\mathrm{H} 1 \mathrm{hESC}$ differentiation in the presence (open bars) or the absence of VEGF (colored bars). Data are presented as mean $\pm S D, n=3$. (E) Day 20 phenotype of the MYB knockout cell lines differentiated in the absence of VEGF. Data are representative of three independent differentiation experiments.

independent early blood cells since these early cells are strongly suppressed by the VEGF removal (Figure 6A and D). Progenitor-independent monocyte/macrophages, granulocytes, and their immature precursors are likely to arise through the conversion of mesodermal or endothelial cells in a process similar to the aforementioned in vitro EHT. MYB inactivation severely affected the progenitorindependent myeloid cells with complete loss of granulocytes on day 20 of the VEGF-deprived differentiation (Figure 6E). Taken together, these results demonstrate that VEGF serves as a major mitogenic factor for the nascent human blood cells.

\section{MYB is required for the development and proliferation of primitive progenitors}

The progenitor defects upon MYB inactivation might be either due to the failure of progenitor proliferation in the methylcellulose assay or the interruption of progenitor development during the primary differentiation of hESC. In order to address this issue, we performed a series of rescue experiments in MYB-null cells using a Tet-On expression system $\mathrm{PB}-\mathrm{iDox}$ that included a PiggyBac transposon vector ${ }^{25}$ bearing $M Y B$ cDNA under the TRE-CMV promoter. One type of the doxycycline (DOX)-inducible vectors contained $m$ Cherry gene reporter attached to $M Y B$ cDNA via an IRES element (Figure 7A and B). In CFU-C colonies that were generated from DOX-activated DKO cells transfected by the PB-MYB-mCherry construct, the red reporter was downregulated at the colony fringes containing more mature cells (Figure 7C). The transgene's downregulation was even more profound than the silencing of MYB-Venus expression in the fringes (Online Supplementary Figure S7A). This observation suggests that the transgene's expression is controlled by post-transcriptional regulatory mechanisms, possibly via microRNA, ${ }^{26}$ and the overexpression of $\mathrm{MYB}$, a typical proto-oncogene, does not cause an uncontrolled proliferation of hematopoietic cells and progenitors. A time-course analysis of the PB- $M Y B$ transgene expression in DOX-treated DKO cells demonstrated stage-specific modulation of $M Y B$ mRNA levels further suggesting the post-transcriptional regulation by microRNA (Online Supplementary Figure S7B).

We have been able to rescue day 6 and day 12 clonogenic progenitors only when the transgenic MYB was induced by DOX both in the primary differentiation of $\mathrm{hESC}$ and the secondary methylcellulose cultures (Figure 7D; Online Supplementary Figure S7C). The most efficient was the recovery of BFU-E $\mathrm{E}^{\mathrm{P}}$ on day 6 (Figure 7D) and myeloid progenitors on day 12 (Online Supplementary Figure S7C) whereas the rescue of CFU-mix progenitors 
was at a very low but detectable level at both stages. These results demonstrated that the gene is required not only for progenitor proliferation but also for their development from differentiated hESC. In addition, the progenitor recovery further evidenced that defects in the development of primitive hematopoietic progenitors were associated with the MYB inactivation rather than nonspecific genomic abnormalities.
We also studied whether the reactivation of MYB in the MYB-null cells could rescue maturating myeloid cells at day 20 of the primary differentiation. As shown in Figure $7 \mathrm{E}$, the continuous expression of $\mathrm{PB}-M Y B$ from day 4 to day 18 has led to a robust recovery of $\mathrm{CD}_{11 \mathrm{~b}^{+}}$and $\mathrm{CD} 11 \mathrm{c}^{+}$monocyte-macrophage cells with strong upregulation of CD86 and HLA-DR. In contrast, only limited recovery of $\mathrm{CD} 6 \mathrm{~b}^{+}$granulocytes was observed on day
A

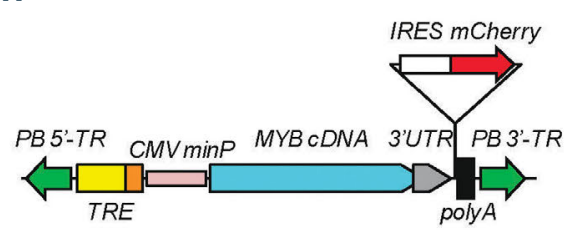

D

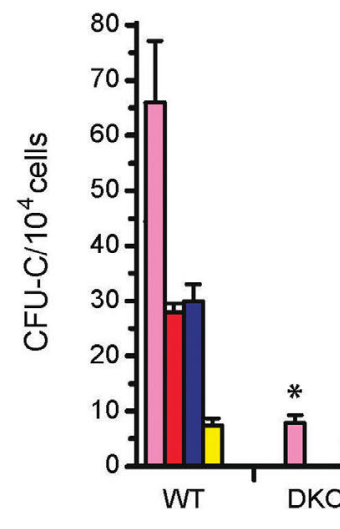

B
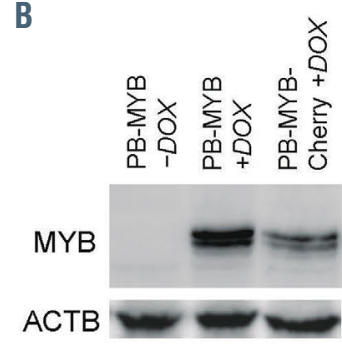

ACTB

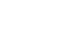

C

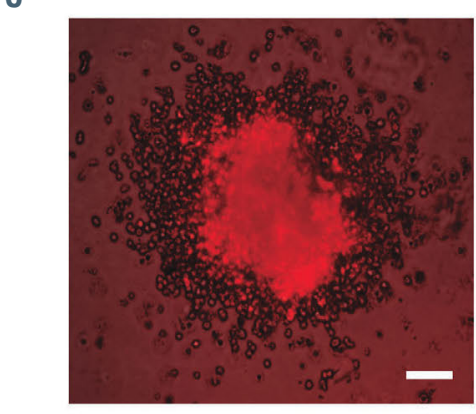

DOX added in primary and secondary culture

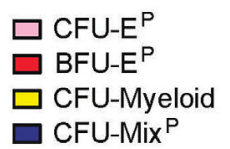

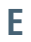
DOX added only
in primary culture
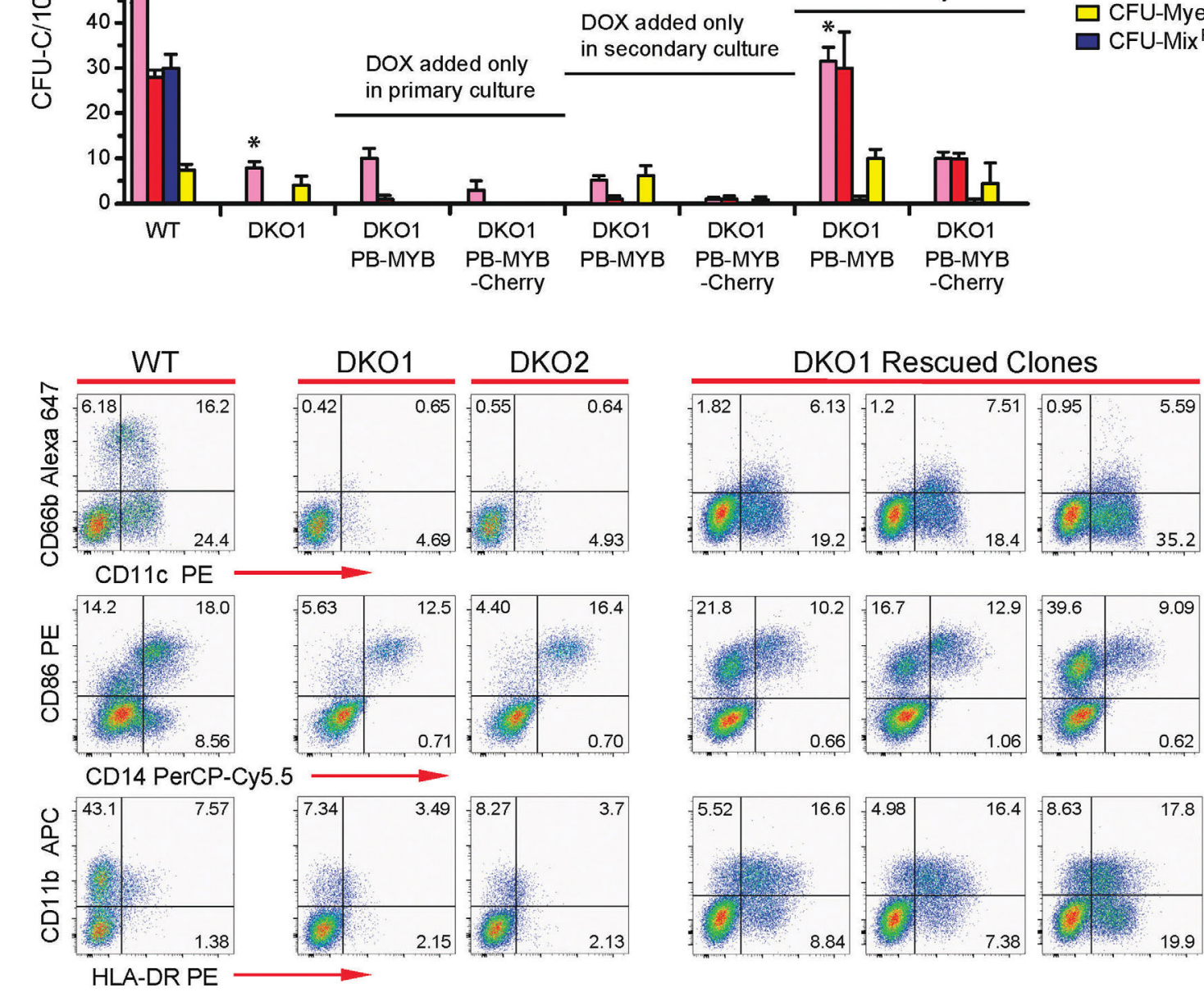

Figure 7. MYB is required for both emergence and proliferation of the primitive clonogenic progenitors. (A) Scheme of the PiggyBac-MYB transposon construct. (B) Western blotting of doxyclycline (DOX)-induced expression of transgenic MYB in day 12 DKO1-PB-MYB and DKO1-PB-MYB-Cherry cells. (C) A representative CFU-mix colony generated by DOX-stimulated day 6 DKO1-PB-MYB-Cherry cells. Scale bar, $100 \mu \mathrm{m}$. (D) Efficient rescue of day 6 clonogenic progenitors is achieved when DOX is added to both primary and secondary stages of hematopoietic differentiation of DKO1-PB-MYB cells. Data are mean \pm standard deviation (SD), $n=3$. ( $E$ ) MYB overexpression in the DKO1-PB-MYB clones during day 4 to day 18 period leads to the accumulation of immature myeloid cells on day 20 . Representative data of three experiments are shown. 
20 , suggesting that the granulocyte differentiation and maturation were inhibited by MYB overexpression that resulted in the accumulation of immature CD11 $\mathrm{c}^{+} \mathrm{CD}_{66 b^{\text {low }}}$ myeloid cells. The negative effect of Myb overexpression on granulocyte maturation has been observed in the mouse progenitor differentiation model. ${ }^{27}$ Taken together with the limited recovery of CFU-mix progenitors, these data suggest that an excess of MYB dysregulates the hESC-derived hematopoietic development.

\section{Discussion}

In this study, we created a model in which the emergence of the early primitive blood cells was effectively uncoupled from the development of the primitive hematopoietic progenitors. The ability of differentiated hESC to generate large numbers of blood cells in the absence of cytokines provided an initial idea that early blood cells do not require the proliferation of clonogenic hematopoietic progenitors for their emergence. We have been unable to detect mRNA of EPO, TPO, G-CSF, GM-CSF, and IL3 in differentiating cultures of hESC up to day 12; thereby the hESC-derived erythro-myeloid progenitors are unlikely to proliferate and produce day 6 primitive blood cells.

The conclusive evidence of progenitor independence of early human blood cells was obtained from our gene ablation studies of $M Y B$. Primitive erythroid and CFUMix ${ }^{\mathrm{P}}$ progenitors failed to develop in the absence of MYB and thus could not be a source of the MYB-independent cells of the erythro-megakaryocyte lineage. These data suggest that the primitive clonogenic progenitors and the first wave of primitive blood cells originate from $\mathrm{HE}$ independently (Figure 8). Our findings contradict the convention that all primitive blood cells are derived from primitive progenitors. ${ }^{1,3,4}$ Despite their MYB-independence the early human blood cells specifically upregulated transcription from $M Y B$ promoters. One possible explanation of this observation is that the absence of the functional MYB protein is compensated by other transcription factors. Alternatively, the MYB expression is an insufficient prerequisite for turning $\mathrm{HE}$ and early blood cells into hematopoietic progenitors. In this view, additional developmental cues select cells for specification into functional progenitors.

We obtained ample evidence that MYB-dependent hematopoietic progenitors belonged to the primitive wave of hematopoiesis. These observations indicate the presence of MYB-dependent primitive erythro-myeloid progenitors (EMP) in the early human hematopoietic development. The issue of definitive versus primitive EMP in humans is disputed ${ }^{28}$ it is possible that both primitive and definitive cohorts of EMP participate in the establishment of human hematopoiesis. One report noticed abnormalities both in primitive and definitive hematopoiesis in a c-myb mutant of the medaka fish. ${ }^{29}$ However, the $c-m y b$ mutation creates a dominant-negative allele that could inhibit the function of the related $a-m y b$ and $b$-myb genes. ${ }^{30}$ Other mouse and zebrafish studies unequivocally denied $\mathrm{Myb} / \mathrm{myb}$ a role in the primitive hematopoiesis.,22 Our data challenged the notion of MYB as a factor that does not have a function in the primitive hematopoiesis. The differences in the developmental role of MYB between small model animals and humans are likely to be associated with the requirement to facilitate the longer proliferation of primitive blood cells in order to sustain the growing needs of human conceptuses.

An important issue to consider is whether our findings are relevant to the onset of hematopoietic development in the human conceptus. Poor quality and scarcity of $3^{\text {rd }}$ week human conceptuses prevent reliable analysis of the early human hematopoietic cells. ${ }^{31}$ Detailed mouse studies, however, suggest that around 300 EryP-CFC on E8. $5^{[1]}$ are unlikely to give rise to about $10^{5}$ erythroblasts in the E9.5 conceptus, ${ }^{32}$ and there should be a progenitor-independent source of the primitive blood cells. Such large

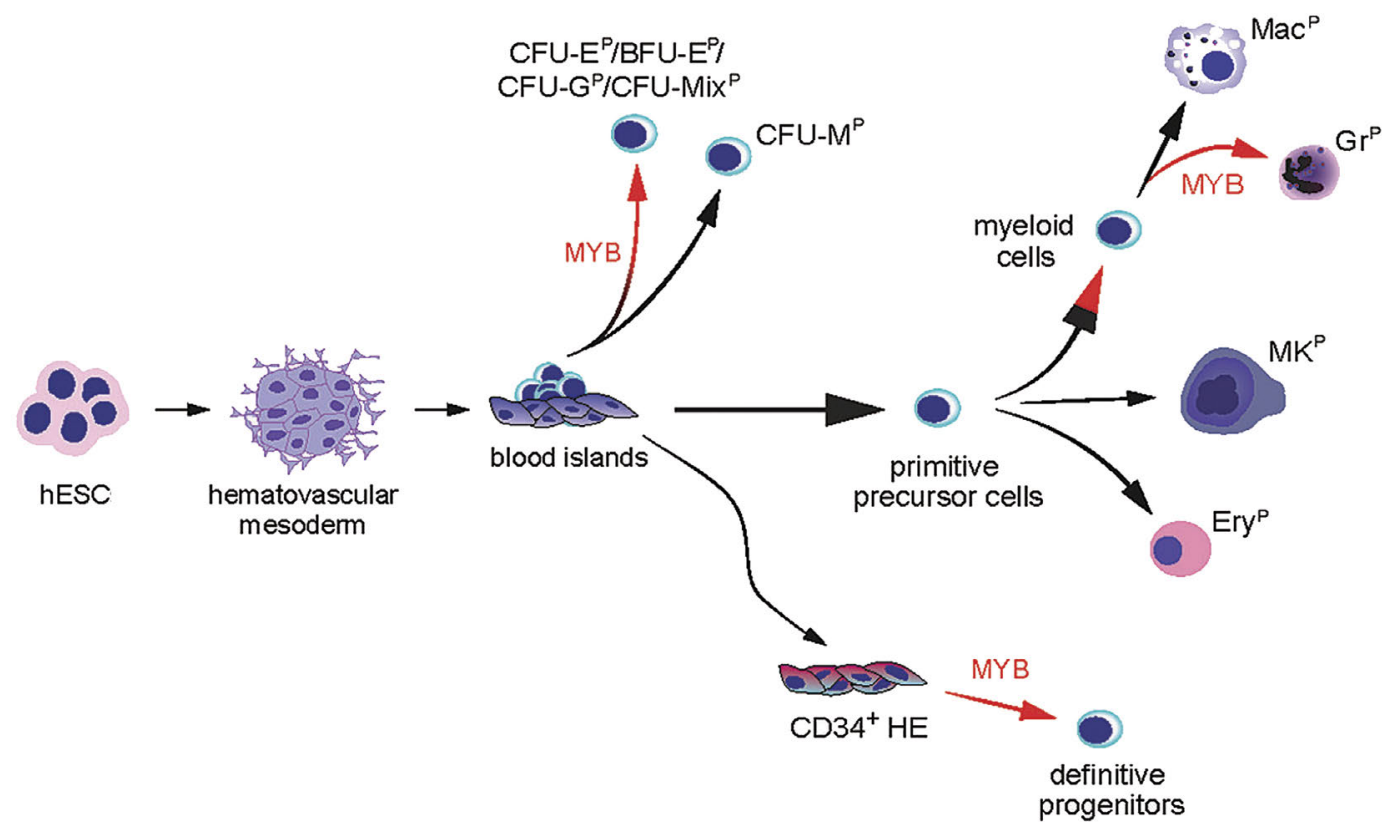

Figure 8. The role of MYB in the human embryonic stem cell-derived hematopoiesis. The MYB gene targeting and the cytokine-free differentiation system revealed progenitor-independent primitive hematopoiesis. Red arrows show MYB-dependent developmental events, black arrows - MYB-independent stages. 
numbers of the primitive cells may be provided by the massive EHT in the yolk sac vasculature.

The detection of the progenitor-independent primitive blood cells was possible only in cytokine-free culture conditions because otherwise, it would be difficult to distinguish progenitor-derived from HE-derived blood cells. Our findings further emphasize the value of the in vitro differentiation of hESC as a practical tool for studying early hematopoiesis in humans.

\section{Disclosures}

No conflicts of interest to disclose.

\section{Contributions}

IMS designed and directed the study; ZSh co-designed the study, created the mutant cell lines, and performed most of the experiments; $C F$ and $H U$ performed a number of CFU-C assays, $q R T-P C R$, and VEGF-independent differentiation; $B Z$ provided the experimental data on Activin/Nodal signaling inhibition; ESF conducted immunocytofluorescent staining; $C W$ prepared cell samples for the RNA-seq analysis; VR, CW, $P V$ performed bioinformatics analyses; IMS wrote the manuscript with input from ESF, ZSh, and PV.

\section{Acknowledgments}

The authors thank Dr. Kenjiro Adachi (MPI Münster) for providing the PiggyBac plasmids, Dr. Guangjin Pan (GIBH CAS) for providing HA hESC line, and Andrew Sonin (MIPT) for assistance with bioinformatics analyses.

\section{Funding}

This work was supported by the National Key R\&D Program of China 2017YFA0103101, Science and Technology Planning Project of Guangdong Province, China 2017B030314056, the National Basic Research Program of China 2015CB964900, the Guangdong Province Leading Talent Program 2014-2018 (to IMS).

\section{References}

1. Palis J, Robertson S, Kennedy M, Wall C, Keller G. Development of erythroid and myeloid progenitors in the yolk sac and embryo proper of the mouse. Development. 1999;126(22):5073-5084.

2. Ferkowicz MJ, Yoder MC. Blood island formation: longstanding observations and modern interpretations. Exp Hematology. 2005;33(9):1041-1047.

3. Wong PM, Chung SW, Reicheld SM, Chui $\mathrm{DH}$. Hemoglobin switching during murine embryonic development: evidence for 2 populations of embryonic erythropoietic progenitor cells. Blood. 1986;67(3):716-721.

4. Porcher C, Chagraoui $H$, Kristiansen MS. SCL/TAL1: a multifaceted regulator from blood development to disease. Blood. 2017;129(15):2051-2060.

5. Kingsley PD, Malik J, Fantauzzo KA, Palis J. Yolk sac-derived primitive erythroblasts enucleate during mammalian embryogenesis. Blood. 2004;104(1):19-25.

6. Ramsay RG, Gonda TJ. MYB function in normal and cancer cells. Nat Rev Cancer. 2008;8(7):523-534

7. Tober J, McGrath KE, Palis J. Primitive erythropoiesis and megakaryopoiesis in the yolk sac are independent of c-myb. Blood. 2008;111(5):2636-2639.

8. Mucenski ML, McLain $\mathrm{K}$, Kier $\mathrm{AB}$, et al. A functional c-myb gene is required for normal murine fetal hepatic hematopoiesis. Cell. 1991;65(4):677-689.

9. Vanhee S, De Mulder K, Van Caeneghem Y, et al. In vitro human embryonic stem cell hematopoiesis mimics MYB-independent yolk sac hematopoiesis. Haematologica ,2015;100(2):157-166.

10. Buchrieser J, James W, Moore MD. Human induced pluripotent stem cell-derived macrophages share ontogeny with MYBindependent tissue-resident macrophages. Stem Cell Rep. 2017;8(2):334-345

11. Jacobs SM, Gorse KM, Westin EH. Identification of a second promoter in the human c-myb proto-oncogene. Oncogene. 1994;9(1):227-235.

12. Thompson MA, Flegg $R$, Westin $E H$, Ramsay RG. Microsatellite deletions in the c-myb transcriptional attenuator region associated with over-expression in colon tumor cell lines. Oncogene. 1997; 14(14):1715-1723.

13. Salvagiotto G, Burton S, Daigh CA, Rajesh D, Slukvin II, Seay NJ. A defined, feeder free, serum-free system to generate in vitro hematopoietic progenitors and differentiated blood cells from hESCs and hiPSCs. PLoS One. 2011;6(3):e17829.

14. Niwa A, Heike T, Umeda K, et al. A nove serum-free monolayer culture for orderly hematopoietic differentiation of human pluripotent cells via mesodermal progenitors. PLoS One. 2011;6(7):e22261.

15. Uenishi G, Theisen D, Lee J-H, et al. Tenascin $\mathrm{C}$ promotes hematoendothelial development and T lymphoid commitment from human pluripotent stem cells in chemically defined conditions. Stem Cell Reports. 2014;3(6):1073-1084.

16. Malik J, Kim AR, Tyre KA, Cherukuri AR, Palis J. Erythropoietin critically regulates the terminal maturation of murine and human primitive erythroblasts Haematologica. 2013;98(11):1778-1787

17. Kennedy M, Awong G, Sturgeon CM, et al. T lymphocyte potential marks the emergence of definitive hematopoietic progenitors in human pluripotent stem cell differentiation cultures. Cell Rep. 2012 ; 2(6):1722-1735

18. Sturgeon CM, Ditadi A, Awong G, Kennedy M, Keller G. Wnt signaling controls the specification of definitive and primitive hematopoiesis from human pluripotent stem cells. Nat Biotechnol. 2014;32(6):554-561.

19. Scheicher R, Hoelbl-Kovacic A, Bellutti F, et al. CDK6 as a key regulator of hematopoietic and leukemic stem cell activation. Blood. 2015;125(1):90-101.

20. Bunz F, Dutriaux C, Lengauer C, et al. Requirement for p53 and p21 to sustain G2 arrest after DNA damage. Science. 1998;282(5393):1497-1501.

21. Bluteau O, Langlois T, Rivera-Munoz P, et al. Developmental changes in human megakaryopoiesis. J Thromb Haemost. 2013;11(9):1730-1741.
22. Soza-Ried C, Hess I, Netuschil N, Schorpp $\mathrm{M}$, Boehm T. Essential role of c-myb in definitive hematopoiesis is evolutionarily conserved. Proc Natl Acad Sci USA 2010;107(40):17304-17308

23. Clarke D, Vegiopoulos A, Crawford A, Mucenski M, Bonife, C, Frampton J. In vitro differentiation of c-myb-/- ES cells reveals that the colony forming capacity of unilineage macrophage precursors and myeloid progenitor commitment are cMyb independent. Oncogene. 2000; 19(30):3343-3351.

24. Sturgeon CM, Ditadi A, Clarke RL, Keller G. Defining the path to hematopoietic stem cells. Nat Biotechnol. 2013;31(5):416-418.

25. Adachi K, Nikaido I, Ohta $\mathrm{H}$, et al Context-dependent wiring of Sox2 regulatory networks for self-renewal of embryonic and trophoblast stem cells. Mol Cell. 2013;52(3):380-392

26. Wang X, Angelis N, Thein SL. MYB - a regulatory factor in hematopoiesis. Gene. 2018;665:6-17.

27. Bies J, Mukhopadhyaya R, Pierce J, Wolff L. Only late, non-mitotic stages of granulocyte differentiation in $32 \mathrm{Dcl} 3$ cells are blocked by ectopic expression of murine cmyb and its truncated forms. Cell Growth Differ. 1995;6(1):59-68.

28. Ivanovs A, Rybtsov S, Ng ES, Stanley EG Elefanty AG, Medvinsky A. Human haematopoietic stem cell development: from the embryo to the dish Development. 2017;144(13):2323-2337.

29. Moriyama A, Inohaya K, Maruyama K, Kudo A. Bef medaka mutant reveals the essential role of c-myb in both primitive and definitive hematopoiesis. Dev. Biol. 2010;345(2):133-143.

30. Lipsick JS. The c-MYB story - is it definitive? Proc Natl Acad Sci USA. 2010; 107(40):17067-17068.

31. Tavian M, Peault B. Embryonic development of the human hematopoietic system. Int J Dev Biol. 2005;49(2-3):243-250.

32. McGrath KE, Koniski AD, Malik J, Palis J. Circulation is established in a stepwise pattern in the mammalian embryo. Blood. 2003;101(5):1669-1676. 